\title{
Evolution in the practice of pharmacy—not a revolution!
}

\author{
Glen J. Pearson
}

I $\mathrm{n}$ an era of rapid change in health care delivery, the pharmacy profession is experiencing significant growth and development. Although pharmacists represent a traditional health profession with ancient roots, they are often viewed with considerable ambiguity and uncertainty by those outside of the profession.

Traditionally, pharmacy was regarded as a transitional discipline between the health and chemical sciences and as a profession charged with ensuring the safe use of medication. In the early Igoos, pharmacists fulfilled the role of apothecary - preparing drug products secundum artem (according to the art) for medicinal use. By the I950s, large-scale manufacturing of medicinal products by the pharmaceutical industry, and the introduction of prescription-only legal status for most therapeutic agents, limited the role of pharmacists to compounding, dispensing and labelling prefabricated products. In response, by the mid-I96os pharmacists had evolved toward a more patient-oriented practice and developed the concept of clinical pharmacy. This marked the beginning of a period of rapid transition that was characterized by an expansion and integration of professional functions, as well as increased professional diversity and closer interaction with physicians and other health care professionals. ${ }^{1-3}$ By the early I9gos the pharmaceutical care model was adopted to emphasize that the role of the pharmacist involves "the responsible provision of drug therapy for the purpose of achieving definite outcomes that improve a patient's quality of life." ${ }^{2}$ To varying degrees across the spectrum of practice environments and specialization in pharmacy, pharmacists are currently recognized as drug experts whose role is to work in collaboration with patients, physicians and other health care professionals to optimize medication management to produce positive health outcomes. ${ }^{2}$

The current transition involves an expansion of the scope of pharmacy practice and, in some jurisdictions, the assumption of the authority to prescribe medications in defined situations. Various prescribing models for pharmacists have previously been developed internationally ${ }^{4,5}$ and have been advocated for within Canadian hospitals. ${ }^{6}$ In the United States, for example, the federal government (Veterans Affairs and Indian Health Service) and at least 40 individual states currently have regulations that authorize collaborative drug therapy management by pharmacists. ${ }^{7}$ In the United Kingdom, supplementary prescribing by pharmacists was approved in 200I. The collaborative drug therapy management and supplementary prescribing models both require partnerships or collaborations between physicians and pharmacists (dependent prescribing model). In April 2006, the Department of Health in the United Kingdom added independent prescribing rights to the official scope of practice of pharmacists. In Canada, pharmacists in some jurisdictions have had

the ability to independently prescribe emergency contraception (now a Schedule II product), but not other prescription medications, since early 2000.

In Alberta, the approval of the Pharmacists Profession Regulation to the Health Professions Act (May 2006) has resulted in an expanded scope of practice for pharmacists, including the privilege to prescribe Schedule I drugs and blood products and to administer medications for subcutaneous and intramuscular injection. The Pharmacy and Drug Act (October 2006) specifically defines the new standards for pharmacy practice. Current legislative changes in Alberta, a province recognized as a leader in health care reform, have been disputed by the medical profession because of "confusion and accusations of conflict of interest."

Pharmacist prescribing, as legislated in Alberta, is substantially different from what is currently understood as prescribing among physicians and other health professions. Primarily, pharmacists have the option to individually decide whether or not they will adopt prescribing authority into their practice. The Alberta pharmacist prescribing model identifies 2 different categories of prescribing.

The first category-"adapting a prescription"-defines the type of prescribing that is most widely applicable to pharmacists. The Alberta College of Pharmacists requires that pharmacists complete an orientation and a continuing education program. In this category of prescribing, both the patient assessment and decision to implement drug therapy is made by a physician or other health care professional. The authorized pharmacist, who has obtained the patient's informed consent, is permitted to make changes to the original prescription but not to prescription renewals. Generic or therapeutic substitutions or changes to the dose or form of drug therapy are allowed as required to satisfy the unique pharmacotherapeutic needs of the patient. After adapting a prescription, the pharmacist is obligated to notify the original prescriber of the rationale for the modifications. The pharmacist then becomes the legal prescriber of the adapted prescription and accepts the associated responsibility. This type of prescribing also grants authority to pharmacists to provide interim prescription refills if the pharmacist determines that it is important to the health of the patient to continue therapy. As the legal prescriber, the pharmacist accepts the responsibility for the refill prescription but must refer the patient to the original prescriber for reassessment and evaluation of the need to continue drug therapy.

The second category of the pharmacist prescribing model is referred to as "initiating/managing drug therapy." This type of prescribing will be limited to pharmacists on the clinical register of the Alberta College of Pharmacists who have successfully completed a 5 -step process to demonstrate the requisite competencies within the context of professional education 
and training, experience, collaborative relationships and practice setting. In this category, authorized pharmacists will be permitted to assess patients and to determine the need to initiate drug therapy. More commonly, pharmacists will work in collaboration with physicians and other health care professionals but will assume responsibility for the management of drug therapy required by the patient. A patient may be referred to an authorized pharmacist by a physician or other health care professional for the purpose of selecting the appropriate drug, dose and dosage form required to treat the condition (referred to as comprehensive drug therapy management). These pharmacists may also assume the responsibility for ongoing drug therapy (monitoring, adjusting, maintaining or initiating drug therapy) for chronic diseases. All prescribing decisions in this category are made under the authority and responsibility of the pharmacist as the legal prescriber.

Not surprisingly, other Canadian provinces are also pursuing expanded scopes of practice for pharmacists. In October 2006 the Saskatchewan College of Pharmacists released a consultation paper proposing prescribing authority for pharmacists in a "collaborative practice framework." In Manitoba the Pharmaceutical Act (Bill 4I), approved in December 2006, provides pharmacists with the authority to prescribe drugs and to order diagnostic tests; however, the regulations for this expanded scope of practice and their effective date are still to be determined. ${ }^{9}$ The British Columbia Pharmacy Association issued a position statement on Jan. 5, 2007, in support of the principle of pharmacist prescribing, but to date the province has not developed any legislation or standards that would permit this practice. ${ }^{10}$

Pharmacist prescribing is scheduled to begin in Alberta on Apr. I, 2007, and will be under intense scrutiny from physicians, other health care professionals and patients. Critics of this advance in the practice of pharmacy should be reassured that, under the proposed standards, it will not be permitted for a prescribing pharmacist to also dispense the product, unless no reasonable alternative is available. This separation between prescribing and dispensing is also required of physicians to ensure patient safety and to prevent any potential conflict of interest. The goals of pharmacist prescribing in Alberta are commendable: improved access to drug therapy, optimized patient outcomes, minimized redundancy and inter- ruptions in the delivery of health care services, and increased collaboration and synergy between pharmacists, physicians and other health care professionals. It is expected that these changes will result in improved health outcomes by optimizing drug therapy for patients in an enhanced health care system. Although these goals are grand, it is anticipated that the Alberta pharmacists who are authorized under the regulations to adopt a prescribing role in their practice will be successful. An orderly transition and constructive evaluation of this new role for pharmacists will be necessary to convince skeptics of the explicit benefits for patient care achieved by the Alberta model for pharmacist prescribing and for future Canadian models.

This article has been peer reviewed.

Glen Pearson is with the Division of Cardiology, University of Alberta, Edmonton, Alta.

Competing interests: None declared.

\section{REFERENCES}

I. Adamcik BA, Ransford HE, Oppenheimer PR, et al. New clinical roles for pharmacists: a study of role expansion. Soc Sci Med I986;23:1187-200.

2. Hepler CD, Strand LM. Opportunities and responsibilities in pharmaceutical care. Am J Pharm Educ 1989;53(suppl):S7-15.

3. Holland RW, Nimmo CM. Transitions, part I: beyond pharmaceutical care. Am J Health Syst Pharm I999;56:1758-64.

4. Emmerton L, Marriot J, Bessell T, et al. Pharmacists and prescribing rights: review of international developments. J Pharm Pharm Sci 2005;8:217-25.

5. Pearson GJ, Yuksel N, Card D, et al. Task Force on Pharmacist Prescribing. An information paper on pharmacist prescribing within a health care facility. Can $J$ Hosp Pharm 2002;55:56-62.

6. Pearson GJ, Yuksel N, Card D, et al; Task Force on Pharmacist Prescribing. Statement on pharmacist prescribing. Can J Hosp Pharm 2002;55:55.

7. Status of collaborative drug therapy management in the United States, March 2004. Am J Health Syst Pharm 2004;61:I609-Io.

8. Priest A. Alberta pharmacists may get prescribing powers. $C M A J$ 2006;175:463-4.

9. Manitoba Society of Pharmacists. Bill $4 \mathrm{I}$ passed with pharmacists retaining voting rights. MSP Member Update 2006;4:I. Available: www.msp.mb.ca/newsletter/ newsletter_4-31.htm (accessed 2007 Mar 8).

Io. British Columbia Pharmacy Association. Pharmacist prescribing position statement. Available: www.bcpharmacy.ca/press_room/documents/PositionStatementPharmacistPrescribing.pdf (accessed 2007 Mar 8).

Correspondence to: Dr. Glen J. Pearson, Division of Cardiology, University of Alberta, Suite $2 C_{2}$ Walter C. Mackenzie Health Sciences Centre, 844O-II2th St., Edmonton AB T6R 3S8; fax 780 407-I496; glenpearson@capitalhealth.ca 